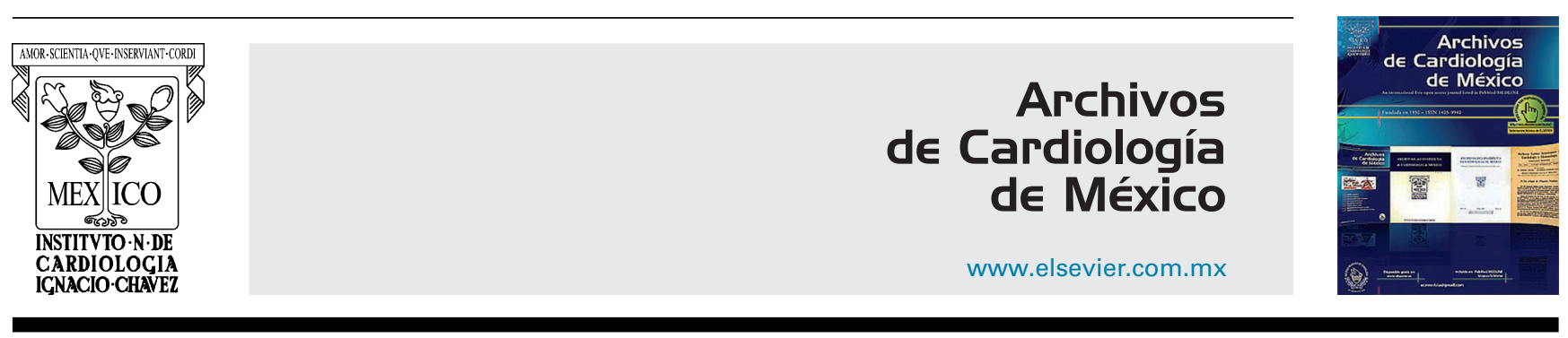

CARTAS CIENTÍFICAS

\section{Upgrade a estimulación tri-ventricular desde estimulación biventricular en un paciente no respondedor a terapia de resincronización cardíaca en fibrilación auricular. ¿Una alternativa terapéutica viable?}

\section{Upgrading to triple-site ventricular stimulation from conventional cardiac resynchronization therapy in a non-responder patient in atrial fibrillation. A feasible therapeutic option?}

\section{Sr. Editor:}

La terapia de resincronización cardíaca (TRC) se ha convertido en uno de los tratamientos con más éxito en los últimos años en pacientes con insuficiencia cardíaca. Sin embargo, aproximadamente un tercio de los pacientes no responden a esta terapia.

Presentamos el caso de un paciente de 82 años portador de marcapasos definitivo bicameral en 1994 por disfunción sinusal. El ECG en el momento del implante muestra un ritmo sinusal a $40 \mathrm{lpm}$ con QRS de $85 \mathrm{~ms}$ y eje normal. En fibrilación auricular (FA) permanente desde 1998. En 2005 se diagnostica de miocardiopatía dilatada con depresión de la función sistólica del ventrículo izquierdo (VI) (FEVI: 29\%) e insuficiencia mitral moderada. Se optimiza el tratamiento farmacológico y se realiza un cateterismo cardíaco que descarta enfermedad coronaria. El porcentaje de estimulación del ventrículo derecho (VD) es del 65\%. El paciente está estable, en clase funcional ॥ de la New York Heart Association (NYHA) hasta 2011 en que se indica un Upgrade a estimulación biventricular por empeoramiento funcional, implantándose un electrodo Medtronic Attain Ability ${ }^{\circledast} 4196$ en una vena posterolateral en posición medio-apical en oblicua anterior derecha (OAD) y en posición $4 \mathrm{~h}$ en oblicua anterior izquierda (OAI). La posición más apical del electrodo del VI se debe a la inestabilidad del electrodo en una posición más basal de la vena posterolateral. Se optimiza el intervalo VI-VD a $-30 \mathrm{~ms}$, según media de integral velocidad tiempo en tracto de salida del VI. Se objetiva una reducción ligera del complejo QRS (fig. 1). En 2014 se realiza una ablación de nodo $\mathrm{AV}$, por bajo porcentaje de estimulación del VI (89\%). El paciente no mejora en su situación funcional. En enero de 2016 ingresa por IC. Se documenta una insuficiencia mitral severa excéntrica, disfunción sistólica del VI severa y asincronía intraventricular izquierda (FEVI: 25\%). El porcentaje de estimulación del VI es del 99\%, el umbral de estimulación es $1.0 \mathrm{v}$ a $0.5 \mathrm{~ms}$, y solamente destaca la posición medio-apical del electrodo del $\mathrm{VI}$ como causa de la no respuesta a la TRC.

Se discuten las opciones terapéuticas que incluyen la extracción del electrodo del VI y reimplante en una vena anterior, implante de un dispositivo de cierre mitral percutáneo y la realización de un Upgrade a estimulación tri-ventricular, y se define la región anterolateral basal como objetivo del nuevo electrodo de estimulación del VI. Debido a la dificultad de garantizar la estimulación percutánea del VI, tras la extracción de un electrodo, que ha permanecido en el interior del seno coronario 5 años, se decide por la opción de estimulación tri-ventricular. Como el paciente está en FA se utiliza el puerto auricular para el nuevo electrodo del VI.

En marzo de 2016 se implanta un electrodo VI Medtronic Attain Ability ${ }^{\circledR}$ plus 4296 en vena anterolateral en posición $2 \mathrm{~h}$ en OAl, en posición basal en OAD (fig. 2A y 2B) con dibujo explicativo de la posición de los electrodos (fig. 2C). El generador es un Medtronic VIVA ${ }^{\circledast}$ CRT-P en modo de estimulación DDDR, AV estimulado y sensado es $30 \mathrm{~ms}$ (valor menor programable de AV en este modelo). El cegamiento ventricular postestimulación auricular es de 30 ms para detección ventricular bipolar (valor no programable). No se observa con esta programación inhibición de estimulación ventricular ni la activación de la estimulación ventricular de seguridad que llevaría a un AV no deseado. El QRS es de $155 \mathrm{~ms}$ (fig. 1).

A los 3 meses de seguimiento, el paciente está en clase funcional ॥ de la NYHA, con reducción de los volúmenes del VI, mejoría en la FEVI hasta el 33\%, y de la asincronía mecánica del $\mathrm{VI}$ y reducción de la insuficiencia mitral por la disminución de la asincronía interpapilar (fig. 2D y E).

El concepto de estimulación multisitio se basa en la hipótesis de que, estimulando en múltiples puntos del ventrículo, mejora el resultado de la TRC. Se han desarrollado 2 modalidades: la primera utiliza 2 electrodos que estimulan en VD y uno en VI. La segunda utiliza 2 electrodos en VI en 2 venas tributarias del seno coronario separadas entre sí. 


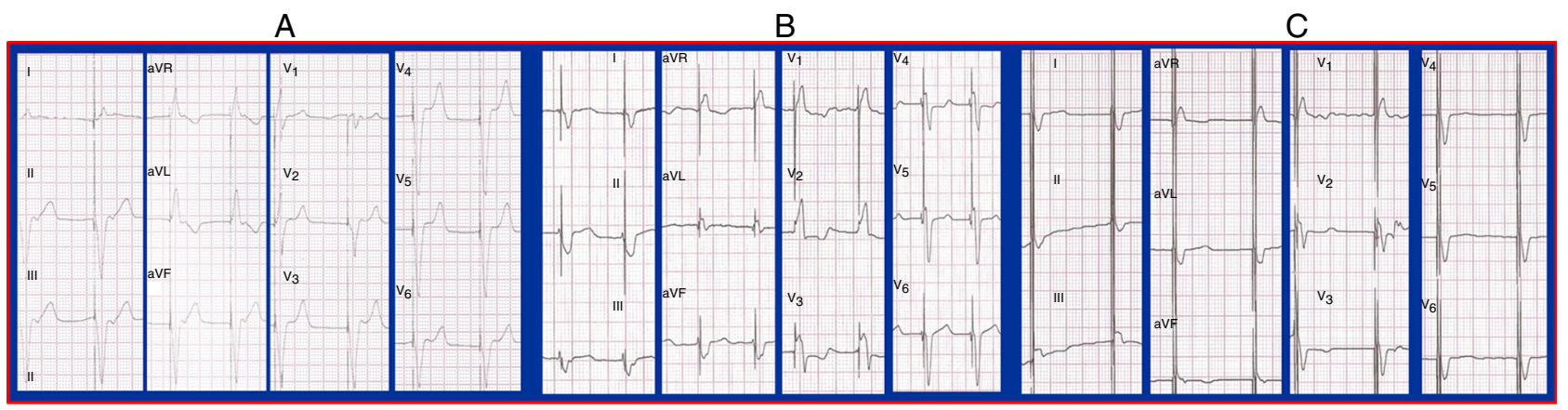

Figura 1 A) Fibrilación auricular con ritmo ventricular estimulado desde ápex de VD. Morfología de QRS similar a BRIHH y eje izquierdo. QRS: $176 \mathrm{~ms}$. B) ECG estimulación biventricular. QRS: $166 \mathrm{~ms}$. C) ECG estimulación tri-ventricular. QRS: $155 \mathrm{~ms}$.

Esta última modalidad ha sido evaluada en estudios hemodinámicos agudos con resultados no concordantes. Pappone et al. demostraron una mejoría en la dP/dt VI, presión de pulso y presión telediastólica del VI con estimulación tri-ventricular ${ }^{1}$. Sin embargo, Padeletti et al. no observaron mejoría en la estimulación tri-ventricular cuando el primer electrodo del VI estaba posicionado de forma óptima ${ }^{1}$. En otro estudio, Ginks et al., mostraron beneficio de la estimulación tri-ventricular solamente cuando había una cicatriz posterolateral ${ }^{1}$.

La viabilidad de la estimulación con 2 electrodos en el VI se alcanza en el 85-95\% en los estudios clínicos, y los resultados a medio plazo son alentadores ${ }^{2}$. En el estudio TRIP-HF ${ }^{3}$, que aleatorizaron a 42 pacientes con FA permanente e indicación de TRC en un diseño cruzado de estimulación biventricular-tri-ventricular, los pacientes en estimulación tri-ventricular no mejoraron significativamente en la clase funcional de la NYHA, ni tampoco en el test de los $6 \mathrm{~min}$ caminando, ni en la calidad de vida respecto a los pacientes en estimulación biventricular, pero sí lo hicieron en la FEVI y el diámetro telesistólico del VI a 3 meses de seguimiento. El estudio TRUST CRT ${ }^{4}$, aleatorizaron a 98 pacientes a estimulación tri-ventricular vs, estimulación biventricular mostrando una mejoría mayor en la clase funcional en el grupo de estimulación tri-ventricular. Recientemente Rogers et al. ${ }^{5}$ han publicado un estudio aleatorizado, doble ciego y
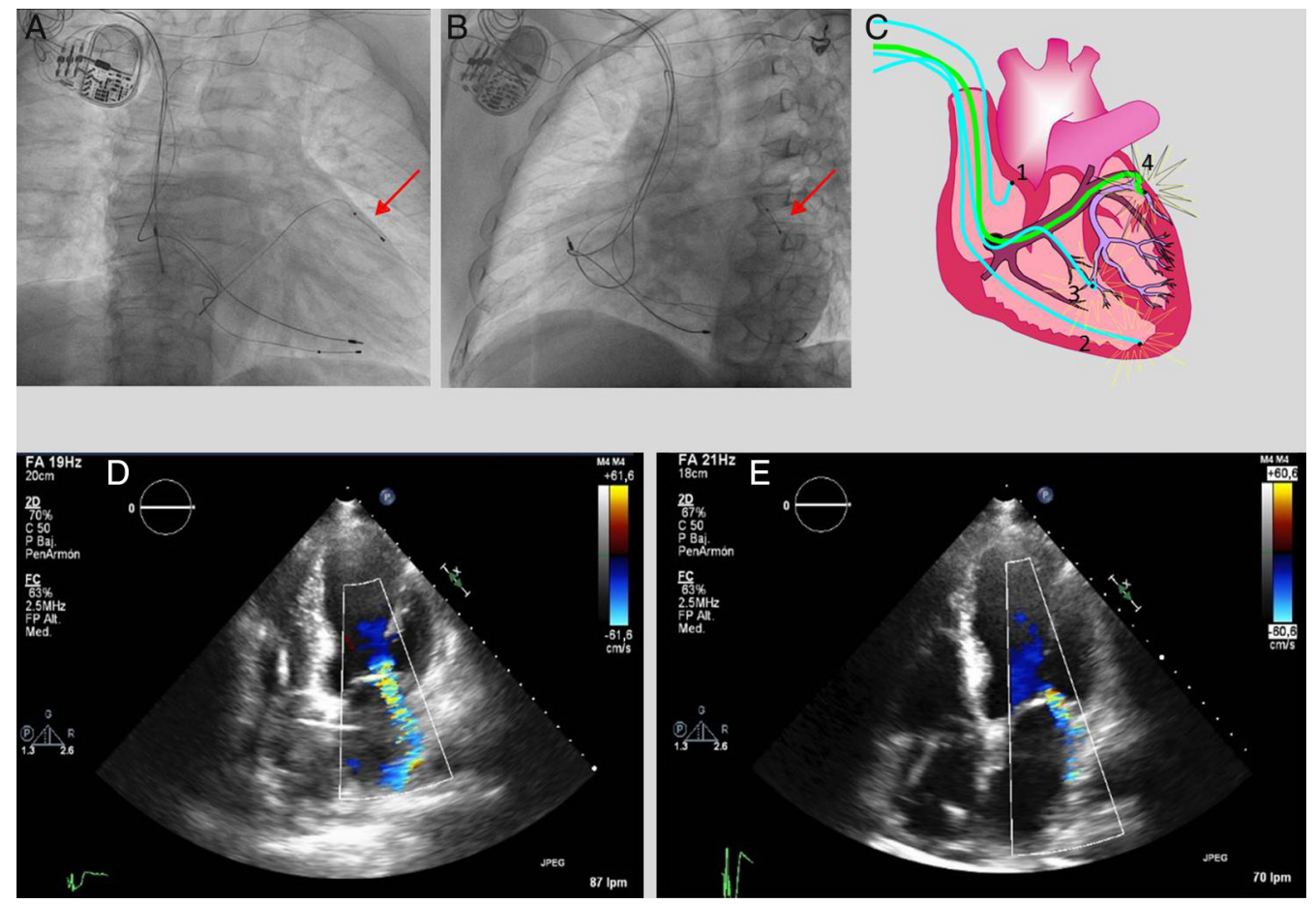

Figura 2 A) Posición del 2. ${ }^{\circ}$ electrodo de VI en vena anterolateral, OAD. B) Posición de 2. ${ }^{\circ}$ electrodo de $\mathrm{VI}$ en vena anterolateral, OAI (flechas rojas). C) Dibujo representativo de la posición de los electrodos en posición OAD: 1: Electrodo auricular no funcionante; 2: Electrodo en ápex de VD; 3: Electrodo en vena posterolateral de VI; 4: Segundo electrodo implantado en vena anterolateral de VI (color verde). Icono luminoso indica punto de estimulación. D) Insuficiencia mitral severa previa a estimulación tri-ventricular: orificio de regurgitación (ORE): $57 \mathrm{~mm}^{2}$, volumen regurgitante (VR): $70 \mathrm{ml}$; VTDVI: $186 \mathrm{ml}$; VTSVI: $142 \mathrm{ml}$ y FEVI: $25 \%$. E) Insuficiencia mitral moderada post estimulación tri-ventricular: ORE: 23 mm². VR: $35 \mathrm{ml}$; VTDVI: $161 \mathrm{ml}$; VTSVI: $108 \mathrm{ml}$; FEVI: $33 \%$. 
cruzado que comparaba la estimulación biventricular con la estimulación tri-ventricular, que a su vez constaba de 2 subgrupos $\left(1^{\circ}\right.$ : estimulación con 2 electrodos en el VD [septo y ápex] y uno en el VI, y 2. ${ }^{\circ}$ : estimulación con un electrodo en el VD y 2 electrodos en el VI). La mejoría en síntomas fue mayor en la estimulación tri-ventricular respecto a la biventricular, pero solamente el $2 .^{\circ}$ grupo de estimulación tri-ventricular (2 electrodos en el VI) presentó un remodelado inverso del VI mayor que el grupo biventricular. Asimismo, se ha publicado una mejor acción anti-arrítmica de la estimulación multisitio (tri-ventricular), respecto a la biventricular estándar con reducción significativa de arritmias ventriculares ${ }^{6}$.

Los estudios presentados incluyen un número pequeño de pacientes, y tienen un seguimiento corto. Teniendo en cuenta que el remodelado inverso del VI puede producirse más allá de un año desde el implante, es necesario evaluar a más largo plazo esta estrategia.

En el momento actual, la estimulación multisitio se puede realizar como una estimulación multipolar, con un electrodo cuadripolar, que permite seleccionar diferentes configuraciones de estimulación dentro de la vena, para obtener el mejor rendimiento hemodinámico. Sin embargo, si el remodelado del VI es muy grande, o si la posición del electrodo no es la óptima, o si está en un área de cicatriz, es posible que esta estrategia no sea suficiente para mejorar la asincronía intraventricular izquierda. En el caso que presentamos no hemos comparado la estrategia de estimulación triventricular con estimulación biventricular, utilizando solo el electrodo anterolateral del VI si bien experimentalmente, la estimulación multipunto en el VI parece superior a la estimulación en un único punto ${ }^{7}$.

La estimulación multisitio con 2 electrodos en el VI parece una técnica viable, que consigue mayor remodelado ventricular inverso ${ }^{2}$. Sin embargo, se necesitan más estudios prospectivos que evalúen a largo plazo el beneficio clínico, el remodelado inverso mayor, y su efecto sobre la supervivencia y los posibles efectos adversos. Mientras tanto podría considerarse de forma individualizada off-label en pacientes no respondedores y asincronía mecánica del VI a pesar de la TRC convencional.

\section{Bibliografía}

1. Rinaldi C, Burri $H$, Thibault $B$, et al. A review of multisite pacing to achieve cardiac resynchronization therapy. Europace. 2015;17:7-17.

2. Anselme F, Bordachar P, LucPasquié J, et al. Safety, feasibility, and outcome results of cardiac resynchronization with triple-site ventricular stimulation compared to conventional cardiac resynchronization. Heart Rhythm. 2016;13:183-9.

3. Leclercq C, Gadler F, Kranig W, et al. A randomized comparison of triple-site versus dual-site ventricular stimulation in patients with congestive heart failure. J Am Coll Cardiol. 2008;51:1455-62.

4. Lenarczyk R, Kowalski O, Kukulski T, et al. Mid-term outcomes of triple-site vs. conventional cardiac resynchronization therapy: A preliminary study. Int J Cardiol. 2009;133:87-94.

5. Rogers $P$, Lambiase $P$, Lowe $M$, et al. A randomized double-blind crossover trial of triventricular versus biventricular pacing in heart failure. Eur J Heart Fail. 2012;14:495-505.

6. Ogano M, Iwasaki Y, Tanabe J, et al. Antiarrhythmic effect of cardiac resynchronization therapy with triple-site biventricular stimulation. Europace. 2013;15:1491-8.

7. Ploux S, Strik M, van Hunnik A, et al. Acute electrical and hemodynamic effects of multisite left ventricular pacing for cardiac resynchronization therapy in the dyssynchronous canine heart. Heart Rhythm. 2014;11:119-25.

Javier Garcia Seara ${ }^{a, *}$, José Luis Martínez Sande ${ }^{a}$, Inés Gómez Otero ${ }^{\mathrm{b}}$, Amparo Martínez Monzonis ${ }^{\mathrm{c}}$, Alfonso Varela Román ${ }^{\mathrm{b}}$ y José Ramón González Juanatey ${ }^{\mathrm{d}}$

a Unidad de Arritmias, Servicio de Cardiología y Unidad Coronaria, Hospital Clínico Universitario de Santiago de Compostela, Santiago de Compostela, La Coruña, España

b Unidad de Insuficiencia Cardíaca, Servicio de Cardiología y Unidad Coronaria, Hospital Clínico Universitario de Santiago de Compostela, Santiago de Compostela, La Coruña, España

c Unidad de Imagen Cardíaca, Servicio de Cardiología y Unidad Coronaria. Hospital Clínico Universitario de Santiago de Compostela, Santiago de Compostela, La Coruña, España

d Servicio de Cardiología y Unidad Coronaria, Hospital Clínico Universitario de Santiago de Compostela, Santiago de Compostela, La Coruña, España

* Autor para correspondencia. Travesía de la Choupana, s/n, 15703 Santiago de Compostela, La Coruña, España; Teléfono: +34636705940.

Correo electrónico: Javier.Garcia.Seara@sergas.es

(J. Garcia Seara).

http://dx.doi.org/10.1016/j.acmx.2016.10.008

$1405-9940$

(c) 2016 Instituto Nacional de Cardiología Ignacio Chávez. Publicado por Masson Doyma México S.A. Este es un artículo Open Access bajo la licencia CC BY-NC-ND (http:/ / creativecommons.org/licenses/bync-nd/4.0/). 